\title{
Thermoviscoelastic Vibrations of a Micro-Scale Beam Subjected to Sinusoidal Pulse Heating
}

\author{
D. S. Mashat \\ Department of Mathematics, Faculty of Science, King Abdulaziz University, P.O. Box 80203, Jeddah 21589, Saudi \\ Arabia
}

A. M. Zenkour

Department of Mathematics, Faculty of Science, King Abdulaziz University, P.O. Box 80203, Jeddah 21589, Saudi Arabia

Department of Mathematics, Faculty of Science, Kafrelsheikh University, Kafrelsheikh 33516, Egypt

\section{A. E. Abouelregal}

Department of Mathematics, College of Science and Arts, Aljouf University, Al-Qurayat, Saudi Arabia Department of Mathematics, Faculty of Science, Mansoura University, Mansoura 35516, Egypt

\begin{abstract}
(Received 11 September 2016; accepted 1 March 2017)
In this article, we studied the vibrational effects of a viscoelastic micro-scale beam induced by sinusoidal pulse heating based on Euler-Bernoulli beam theory. The formulation of the present problem is applied to the generalized thermoelasticity with phase lags. The deflection, temperature, axial displacement, and bending moment of the micro-scale beam are determined by using the Laplace transform method. The system of governing equations is reduced to a novel six-order thermoelastic differential equation in either deflection or temperature. The numerical results in case of silicon material are presented with the help of Mathematica programming software. Some plots are illustrated to investigate the effects of the phase-lags and the width of the sinusoidal pulse parameters. The effect of the viscosity on the micro-scale beam resonator is also investigated.
\end{abstract}

\section{INTRODUCTION}

The linear viscoelasticity remains an important area of research since most of the solids and polymer-like materials are subjected to a dynamic loading exhibit viscous effect. The stress-strain law for many materials, such as polycrystalline metals and high polymers, can be approximated by the linear viscoelasticity theory. In using different generalized thermoelasticity theories, many authors have considered various linear thermoviscoelastic problems. ${ }^{1-9}$

The classical coupled theory of thermoelasticity is based on a parabolic type of heat conduction equation while the generalized thermoelasticity theory was postulated by Lord and Shulman ${ }^{10}$ as well as Green and Lindsay, ${ }^{11}$ who used a hyperbolic type of heat conduction equation admitting finite speed for thermal disturbances. The theory developed by Lord and Shulman ${ }^{10}$ takes into account only one parameter of relaxation time and has been extended by Dhaliwal and Sherief ${ }^{12}$ in anisotropic media. Another generalization to the theory of thermoelasticity is the dual-phase-lag (DPL) model, which is proposed by Tzou. ${ }^{13,14}$

Laser ultrasound is another innovation; it uses a laser to produce and test ultrasound. ${ }^{15-21}$ It is another branch of ultrasound and includes optics, acoustics, calorifics, electrics, material, physics and so on. Laser ultrasound has numerous points of interest. For example, it is noncontact, nondestructive, quick, precise, and requires minimal effort. In this way, it is typically utilized to distinguish and portray absconds as a part of aviation materials or an aerocraft. Thermally prompted vibration of bars has practical significance in space vehicles, reactor vessels, turbines, and other machine parts are subjected to variable warming. Extremely fast thermal procedures, under the activity of a ultra-short laser heartbeat, are intriguing from the outlook of thermoelasticity, since they require an examination of the coupled temperature and strain fields. This implies the absorption of the laser beat energy results in a limited temperature expand, which thusly causes thermal expansion and creates fast movements in the structure components, in this manner bringing about the ascent of vibrations.

Micro-scale mechanical resonators have high affectability as well as a fast response and are widely used as sensors and modulators. Micro- and nano-mechanical resonators have recently pulled in impressive consideration due to their many significant industrial applications. Perfect analysis of many effects on the features of resonators, such as resonant frequencies and quality influences, is crucial for designing high-performance components. Many authors have considered the vibration and heat exchange procedure of micro-scale beams. ${ }^{22-28}$

The objective of the present work is to determine the components of displacements, stress, temperature, and strain distributions in an isotropic homogeneous thermoviscoelastic thin beam subjected to sinusoidal pulse heating. ${ }^{29-31}$ The problem is solved in context of the theory of generalized thermoviscoelastic with phase lags. An exact solution of the problem is first obtained in Laplace transform space. The inversions of Laplace transforms have been carried out numerically. Numerical results predict finite speeds of propagation for thermoelastic and diffusive waves. To investigate the viscosity and phase lags effects, a comparison is made with the results obtained in the thermoelastic problem. Finally, in taking an appropriate material, the results are presented in a graphical form to 
illustrate the impotency of this problem.

\section{MATHEMATICAL MODELING AND THE FUNDAMENTAL EQUATIONS}

The Kelvin-Voigt model is one of the macroscopic mechanical models commonly used to describe the viscoelastic manner of a material. The model represents the delayed flexible reaction subjected to stress when the deformation is timedependent but recoverable. The dynamic association of the mechanical and thermal fields in solids has pronounced practical applications in current aeronautics, astronautics, atomic reactors, and high-energy element accelerators, for instance we refer to Mishra et al. ${ }^{32}$

We consider a homogenous isotropic thermally conducting Kelvin-Voigt type thermoviscoelastic solid initially at uniform temperature $T_{0}$ and undeformed. The governing equations for a linear isotropic and homogeneous thermoviscoelastic solid in the absence of body forces take the following forms. ${ }^{33}$

The equation of motion has the form

$$
\mu^{*} u_{i, j j}+\left(\mu^{*}+\lambda^{*}\right) u_{j, i j}-\beta^{*} \theta_{, i}=\rho \frac{\partial^{2} u_{i}}{\partial t^{2}} ;
$$

where $u_{i}$ are the components of the displacement vector, $\theta=$ $T-T_{0}, T$ is the absolute temperature of the medium, $T_{0}$ is the reference uniform temperature of the body chosen such that $\left|\theta / T_{0}\right| \ll 1, t$ is time and $\rho$ is the density. The parameters, $\mu^{*}$, $\lambda^{*}$, and $\beta^{*}$, are defined as

$$
\begin{gathered}
\lambda^{*}=\lambda\left(1+\alpha_{1} \frac{\partial}{\partial t}\right) ; \quad \mu^{*}=\mu\left(1+\alpha_{2} \frac{\partial}{\partial t}\right) ; \\
\beta^{*}=\beta\left(1+\beta_{1} \frac{\partial}{\partial t}\right) ; \quad \beta=(3 \lambda+2 \mu) \alpha_{t} ; \\
\beta_{1}=\frac{\left(3 \lambda \alpha_{1}+2 \mu \alpha_{2}\right) \alpha_{t}}{\beta} ;
\end{gathered}
$$

where $\lambda$ and $\mu$ are Lamés constants, $\alpha_{1}, \alpha_{2}$ are the thermoviscoelastic relaxation times, and $\alpha_{t}$ is the coefficients of linear thermal expansion.

The strain-displacement relations are given by

$$
e_{i j}=\frac{1}{2}\left(u_{i, j}+u_{j, i}\right)
$$

The heat conduction equation in the context of generalized thermoelastic with phase lags, is modified to be of the form

$$
K\left(1+\tau_{\theta} \frac{\partial}{\partial t}\right) \theta_{, i i}=\left(\delta+\tau_{q} \frac{\partial}{\partial t}\right)\left[\rho C_{E} \frac{\partial \theta}{\partial t}+\beta^{*} T_{0} \frac{\partial e}{\partial t}-\rho Q\right]
$$

where $e$ is the strain dilatation, $K$ is the thermal conductivity, $C_{E}$ is the specific heat at constant strain, $\tau_{q}$ is the phase-lag of the heat flux, $\tau_{\theta}$ is phase-lag of the temperature gradient $0 \leq \tau_{\theta}<\tau_{q}$, and $Q$ is the heat source per unit volume. The dummy index implies summation.

The constitutive equations have the form

$$
\sigma_{i j}=2 \mu^{*} e_{i j}+\left(\lambda^{*} e_{k k}-\beta^{*} \theta\right) \delta_{i j}
$$

where $\sigma_{i j}$ is the stress tensor and $\delta_{i j}$ is Kronecker's delta function. Equations (1) to (5) respectively describe the coupled

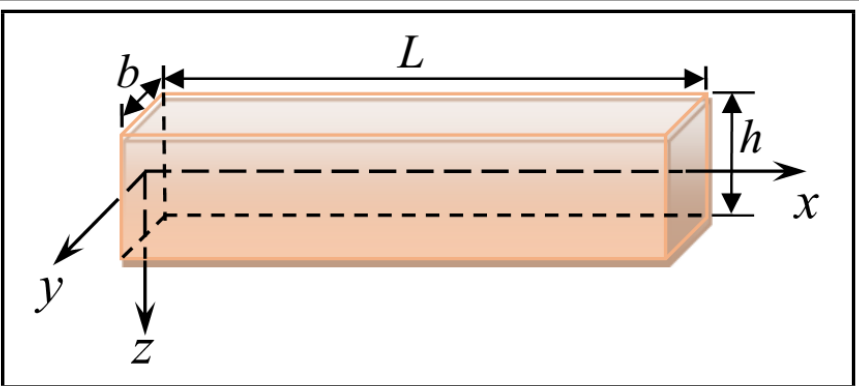

Figure 1. Schematic illustration of the beam set-up.

dynamical thermoviscoelasticity theory, the generalized thermoelasticity theory proposed by Lord and Shulman, the generalized thermoelasticity theory with two relaxation times developed by Green and Lindsay, and Green and Naghdi theory without energy dissipation and dual-phase-lag model for different sets of values of the parameters $\tau_{\theta}$ and $\tau_{q}$.

In Eqs. (1) to (5):

1) If we put $\tau_{\theta}=\tau_{q}=0$, it reduces to the equations of the classical theory of thermoviscoelasticity (CTE).

2) When $\tau_{\theta}=0, \tau_{q} \geq 0$ and $\delta=1$, it reduces to the equations of the generalized thermoviscoelasticity theory with one relaxation time (LS).

3) Putting $\tau_{\theta}=0, \tau_{q}=1$ and $\delta=0$, reduces to the equations of the generalized thermoviscoelasticity theory with two relaxation time (GL).

4) Putting $\tau_{\theta}=0, \tau_{q}=1, \delta=0$, and $K=K^{*}$, ( $K^{*}$ is a material constant characteristic of Green and Naghdi theory), reduces to the equations of the generalized thermoviscoelasticity theory without energy dissipation (GN).

5) If $0 \leq \tau_{\theta}<\tau_{q}$ and $\delta=1$, it reduces to the equations of the generalized thermoviscoelasticity theory with dualphase-lags (DPL).

In absence of viscous effect the viscoelastic relaxations times are zero (i.e., $\alpha_{1}=\alpha_{2}=0$ ).

\section{FORMULATION OF THE PROBLEM}

We consider a homogeneous, thermally conducting, KelvinVoigt type visco-thermoelastic micro-scale beam (Fig. 1) with length $L$, thickness $h$, and width $b$. A Cartesian coordinate system is attached to the beam such that the $x$-coordinate is parallel to the beam axis. The thickness and width directions are parallel to the $y$ - and $z$-axes, respectively, and then structure occupies the domain defined by $0 \leq x \leq L,-b \leq 2 y \leq b$ and $-h \leq 2 z \leq h$.

The beam undergoes bending vibrations of small amplitude about the $x$-axis, such that the deformation is consistent with the linear Euler-Bernoulli theory. The usual Euler-Bernoulli assumption $^{34}$ is made so that any plane cross-section, initially perpendicular to the axis of the beam, remains plane and perpendicular to the neutral surface during bending. Thus, the displacements can be given by

$$
u=-z \frac{\partial w}{\partial x} ; \quad v=0 ; \quad w=w(x, z, t)
$$

where $u$ is the axial displacement and $w$ is the transverse displacement in the $z$ direction (the lateral deflection). The elastic 
stress field in the beam is assumed to be uniaxial; only $\sigma_{x x}$ can attained non-zero values. This assumption is valid for slender Euler-Bernoulli beams and neglects any shear stresses in the vicinity of clamped boundaries. ${ }^{34}$ The constitutive equation reduces to one-dimensional equation yields

$$
\sigma_{x x}=\left(2 \mu^{*}+\lambda^{*}\right) \frac{\partial u}{\partial x}-\beta^{*} \theta .
$$

The flexure moment of the cross-section is given as

$$
M(x, t)=-b \int_{-h / 2}^{h / 2} z \sigma_{x x} d z .
$$

Using Eq. (7) into Eq. (8) to get the flexure moment of beam as follows:

$$
M(x, t)=I\left(2 \mu^{*}+\lambda^{*}\right) z \frac{\partial^{2} w}{\partial x^{2}}+\beta^{*} M_{T}
$$

where $I=b h^{3} / 12$ is moment of inertia of the cross-section and

$$
M_{T}=b \int_{-h / 2}^{h / 2} z \theta(x, z, t) d z
$$

is the thermal moment of the beam.

There is no heat flow across the upper and lower surfaces of the beam, hence $\frac{\partial \theta}{\partial x}=0$ at $z= \pm \frac{h}{2}$. For a very thin beam, it is assumed that the temperature increment varies in terms of a sin function along the thickness direction. That is

$$
\theta(x, z, t)=\phi(x, t) \sin (p z) ; \quad p=\frac{\pi}{h} .
$$

Substituting Eq. (11) into Eq. (10), gives

$$
M_{T}=2 a p^{2} \phi(x, t) .
$$

Substituting from Eq. (12) into Eq. (9), gives

$$
M(x, t)=I\left(2 \mu^{*}+\lambda^{*}\right) z \frac{\partial^{2} w}{\partial x^{2}}+2 a p^{2} \beta^{*} \phi(x, t) .
$$

The equation of motion for free flexural vibrations of the beam is

$$
\frac{\partial^{2} M}{\partial x^{2}}+\rho A \frac{\partial^{2} w}{\partial t^{2}}=0
$$

where $A=b h$, the cross-section area. By substituting Eq. (13) into Eq. (14), we can get the motion equation of the beam as follows:

$$
I\left(2 \mu^{*}+\lambda^{*}\right) \frac{\partial^{4} w}{\partial x^{4}}+\rho A \frac{\partial^{2} w}{\partial t^{2}}+2 a p^{2} \beta^{*} \frac{\partial^{2} \phi}{\partial x^{2}}=0 .
$$

The initial temperature distribution in the beam $T(x, t, 0)=$ $T_{0}$, i.e., $\theta(x, z, t)=0$. Also, for $t=0$, the upper surface $(z=h / 2)$ of the beam is heated uniformly by a laser pulse with non-Gaussian form temporal profile as follows:

$$
\bar{I}(t)=\frac{L_{0}}{t_{p}^{2}} t e^{-\frac{t}{t_{p}}}
$$

where $t_{p}$ is a characteristic time of the laser-pulse, $L_{0}$ is the laser intensity, which is defined as the total energy carried by a laser pulse per unit cross-section of the laser beam. It is clear that the maximum laser intensity decreases as $t_{p}$ increases. In accordance with Royer, ${ }^{18}$ the conduction heat transfer in the beam can be modeled as a one-dimensional problem with an energy source $Q(z, t)$ near the surface, i.e.,

$$
Q(z, t)=\frac{R_{a}}{\delta_{0}} \bar{I}(t) e^{\frac{2 z-h}{2 \delta_{0}}}=\frac{L_{0} R_{a}}{\delta_{0} t_{p}^{2}} t e^{\left(\frac{2 z-h}{2 \delta_{0}}-\frac{t}{t_{p}}\right)}
$$

where $\delta_{0}$ the absorptive depth of heating energy and $R_{a}$ the absorptivity of the irradiated surface. Substituting the EulerBernoulli assumption, namely Eq. (6), and (17) into Eq. (4) gives the thermal conduction equation for the beam.

The heat conduction in Eq. (4), for the five theories CTE, LS, GL, GN, and DPL are described by the following system of partial differential equations for isotropic materials:

$$
\begin{aligned}
& K\left(1+\tau_{\theta} \frac{\partial}{\partial t}\right)\left(\frac{\partial^{2}}{\partial x^{2}}+\frac{\partial^{2}}{\partial z^{2}}\right) \theta= \\
& \quad\left(\delta+\tau_{q} \frac{\partial}{\partial t}\right)\left[\rho C_{E} \frac{\partial \theta}{\partial t}-\beta^{*} T_{0} z \frac{\partial^{3} w}{\partial x^{2} \partial t}-\rho Q\right] .
\end{aligned}
$$

Using Eqs. (11) and (17) into Eq. (18), we get

$$
\begin{aligned}
& \sin (p z)\left(1+\tau_{\theta} \frac{\partial}{\partial t}\right)\left(\frac{\partial^{2}}{\partial x^{2}}-p^{2}\right) \phi=\left(\delta+\tau_{q} \frac{\partial}{\partial t}\right) \\
& {\left[\frac{\rho C_{E}}{K} \sin (p z) \frac{\partial \phi}{\partial t}-\frac{\beta^{*} T_{0}}{K} z \frac{\partial^{3} w}{\partial x^{2} \partial t}-\frac{\rho L_{0} R_{a}}{K \delta_{0} t_{p}^{2}} t e^{\left(\frac{2 z-h}{2 \delta_{0}}-\frac{t}{t_{p}}\right)}\right] .}
\end{aligned}
$$

Multiplying Eq. (19) by $z$ and integrating it with respect to $z$ from $-h / 2$ to $h / 2$, yields

$$
\begin{aligned}
& \left(1+\tau_{\theta} \frac{\partial}{\partial t}\right)\left(\frac{\partial^{2}}{\partial x^{2}}-p^{2}\right) \phi= \\
& \left(\delta+\tau_{q} \frac{\partial}{\partial t}\right)\left[\eta_{0} \frac{\partial \phi}{\partial t}-\beta^{*} \eta_{1} \frac{\partial^{3} w}{\partial x^{2} \partial t}-\eta_{2} f(t)\right]
\end{aligned}
$$

where

$$
\begin{array}{cl}
\eta_{0}=\frac{\rho C_{E}}{K} ; \quad \eta_{1}=\frac{T_{0} h^{3}}{2 p^{2} K} ; & \eta_{2}=\frac{\rho L_{0} R_{a} \delta_{0}}{2 p^{2} K t_{p}^{2}}\left[\frac{h}{2 \delta_{0}}-\right. \\
\left.1+\left(\frac{h}{2 \delta_{0}}+1\right) e^{\frac{-h}{\delta_{0}}}\right] ; & f(t)=t e^{-\frac{t}{t_{p}}} .
\end{array}
$$

\section{DIMENSIONLESS QUANTITIES}

For convenience, we shall use the following nondimensional quantities

$$
\begin{gathered}
\left\{x^{\prime}, w^{\prime}, u^{\prime}, z^{\prime}, L^{\prime}, b^{\prime}\right\}=c_{1} \eta_{0}\{x, w, u, z, L, b\} \\
\left\{t^{\prime}, \tau_{q}^{\prime}, \tau_{\theta}^{\prime}\right\}=c_{1}^{2} \eta_{0}\left\{t, \tau_{q}, \tau_{\theta}\right\} ; \quad \theta^{\prime}=\frac{\beta}{\rho c_{1}^{2}} \theta ; \\
\sigma_{x x}^{\prime}=\frac{1}{\rho c_{1}^{2}} \sigma_{x x} ; \quad Q^{\prime}=\frac{1}{K c_{1}^{2} \eta_{2}^{2} T_{0}} Q ; \quad c_{1}^{2}=\frac{2 \mu+\lambda}{\rho} ;
\end{gathered}
$$

where $c_{1}$ is the speed of propagation of isothermal elastic waves. For simplicity, we drop the dashes of all variables and parameters. The resulting non-dimensional Eqs. (15) and (20) 
are

$$
\begin{aligned}
& \left(1+c_{2}^{2} \frac{\partial}{\partial t}\right) \frac{\partial^{4} w}{\partial x^{4}}+\eta_{3} \frac{\partial^{2} w}{\partial t^{2}}+\eta_{4}\left(1+\beta_{1} \frac{\partial}{\partial t}\right) \frac{\partial^{2} \phi}{\partial x^{2}}=0 \\
& \left(1+\tau_{\theta} \frac{\partial}{\partial t}\right)\left(\frac{\partial^{2}}{\partial x^{2}}-p^{2}\right) \phi= \\
& \left(\delta+\tau_{q} \frac{\partial}{\partial t}\right)\left[\frac{\partial \phi}{\partial t}-\varepsilon \eta_{5}\left(1+\beta_{1} \frac{\partial}{\partial t}\right) \frac{\partial^{3} w}{\partial x^{2} \partial t}-\eta_{6} f(t)\right] \\
& \sigma_{x x}=-z\left(1+\eta_{0} c_{2}^{2} \frac{\partial}{\partial t}\right) \frac{\partial^{2} w}{\partial x^{2}}-\sin (p z)\left(1+\beta_{1} \frac{\partial}{\partial t}\right) \phi
\end{aligned}
$$

where

$$
\begin{array}{ll}
c_{2}^{2}=\frac{\alpha_{1} \lambda+2 \alpha_{2} \mu}{\rho} ; \quad \eta_{3}=\frac{12}{h^{2}} ; & \eta_{4}=\frac{24 p^{2} c_{1}^{5} \eta_{0}}{h^{3}} ; \\
\eta_{5}=\frac{h^{3}}{2 p^{2} c_{1}^{6} \eta_{0}^{4}} ; \quad \eta_{6}=\frac{\beta \eta_{2}}{\rho c_{1}^{4} \eta_{0}^{3}} ; \quad \varepsilon=\frac{\beta^{2} T_{0}}{\rho^{2} C_{E} c_{1}^{2}}
\end{array}
$$

\section{SOLUTION OF THE PROBLEM IN LAPLACE TRANSFORM DOMAIN}

In order to solve the problem, both the initial and boundary conditions should be considered. The initial conditions of the problem are taken as

$$
\begin{aligned}
& \left.w(x, t)\right|_{t=0}=\left.\frac{\partial w(x, t)}{\partial t}\right|_{t=0}=0 ; \\
& \left.\phi(x, t)\right|_{t=0}=\left.\frac{\partial \phi(x, t)}{\partial t}\right|_{t=0}=0 .
\end{aligned}
$$

By applying Laplace transform to Eqs. (23) to (25), we get the field equations in the Laplace transform space as

$$
\begin{gathered}
\frac{d^{4} \bar{w}}{d x^{4}}+A_{1} \bar{w}+A_{2} \frac{d^{2} \bar{\phi}}{d x^{2}}=0 ; \\
\left(\frac{d^{2}}{d x^{2}}-A_{3}\right) \bar{\phi}=-A_{4} \frac{d^{2} \bar{w}}{d x^{2}}-A_{5} \bar{g}(s) ; \\
\bar{\sigma}_{x x}=-\left(1+\eta_{0} c_{2}^{2} s\right) z \frac{d^{2} \bar{w}}{d x^{2}}-\sin (p z)\left(1+\beta_{1} s\right) \bar{\phi} ;
\end{gathered}
$$

where

$$
\begin{array}{rlrl}
A_{1}=\frac{s^{2} \eta_{3}}{1+s c_{2}^{2}} ; & A_{2} & =\frac{\eta_{4}\left(1+\beta_{1} s\right)}{1+s c_{2}^{2}} ; & A_{3}=p^{2}+q \\
q=\frac{s\left(\delta+\tau_{q} s\right)}{1+\tau_{\theta} s} ; & A_{4}=q \eta_{5} \varepsilon\left(1+\beta_{1} s\right) ; & A_{5}=q \eta_{6} \\
\bar{g}(s) & =\left(\frac{t_{p}}{1+t_{p} s}\right)^{2} ;
\end{array}
$$

and an over bar symbol denotes its Laplace transform, $s$ denotes the Laplace transform parameter. The solution of Eqs. (28) and (29) leads to the following differential equation for $\bar{w}$

$$
\frac{d^{6} \bar{w}}{d x^{6}}-a_{1} \frac{d^{4} \bar{w}}{d x^{4}}+a_{2} \frac{d^{2} \bar{w}}{d x^{2}}-a_{3} \bar{w}=0
$$

where the coefficients $a_{1}, a_{2}$, and $a_{3}$ are given by

$$
a_{1}=A_{2} A_{4}+A_{3} ; \quad a_{2}=A_{1} ; \quad a_{3}=A_{1} A_{3} .
$$

The characteristic equation of Eq. (32) is

$$
k^{6}-a_{1} k^{4}+a_{2} k^{2}-a_{3}=0 ;
$$

where $k_{1}^{2}, k_{2}^{2}$, and $k_{3}^{2}$ are the roots of the characteristic equation. These roots are given by ${ }^{21}$

$$
k_{i}^{2}=\frac{1}{3}\left[\psi_{i}\left(2 a_{1}^{3}+27 a_{3}-9 a_{1} a_{2}\right)^{1 / 3}+a_{2}\right], \quad i=1,2,3 ;
$$

where

$$
\begin{gathered}
\left\{\psi_{1}, \psi_{2}, \psi_{3}\right\}=-\frac{1}{2}\left\{2 R_{0},-R_{0}+i \sqrt{3} R_{1},-R_{0}-i \sqrt{3} R_{1}\right\} \\
R_{0}=\frac{\xi^{2}-R_{0}}{\xi} ; \quad R_{1}=\frac{\xi^{2}+R_{2}}{\xi} \\
R_{2}=\frac{3 a_{2}-a_{1}^{2}}{\left(2 a_{1}^{3}+27 a_{3}-9 a_{1} a_{2}\right)^{2 / 3}} \\
\xi=\frac{1}{2}\left(4+4 \sqrt{4 R_{2}^{3}+1}\right)^{1 / 3}
\end{gathered}
$$

Using Eq. (28) in Eq. (29), we get

$$
\bar{\phi}=\frac{-1}{A_{2} A_{3}}\left(\frac{d^{4} \bar{w}}{d x^{4}}-A_{2} A_{4} \frac{d^{2} \bar{w}}{d x^{2}}+A_{1} \bar{w}-A_{2} A_{5} \bar{g}(s)\right) .
$$

Then we can express the general solutions of Eqs. (32) and (37) by the linear combination of the fundamental solutions. That is

$$
\begin{gathered}
\bar{w}=\sum_{j=1}^{3}\left(C_{j} e^{-k_{j} x}+C_{j+3} e^{k_{j} x}\right) ; \\
\bar{\phi}=\sum_{j=1}^{3} H_{j}\left(C_{j} e^{-k_{j} x}+C_{j+3} e^{k_{j} x}\right)+H_{4} ;
\end{gathered}
$$

where

$$
H_{j}=\frac{-1}{A_{2} A_{3}}\left(k_{j}^{4}-A_{2} A_{4} k_{j}^{2}+A_{1}\right) ; \quad H_{4}=\frac{A_{5} \bar{g}(s)}{A_{3}} ;
$$

are parameters depending on $s$. Thus, Eqs. (38) and (39) constitute a complete set of governing equations for the homogenous isotropic Kelvin-Voigt type thermoviscoelastic beam. In addition, these equations can also be supplemented with appropriate initial and boundary conditions of the relevant problem to be modelled. The displacement after using Eq. (38) takes the form

$$
\bar{u}=-z \frac{d \bar{w}}{d x}=z \sum_{j=1}^{3} k_{j}\left(C_{j} e^{-k_{j} x}-C_{j+3} e^{k_{j} x}\right) ;
$$

and the strain will be

$$
\bar{e}=-z \frac{d^{2} \bar{w}}{d x^{2}}=-z \sum_{j=1}^{3} k_{j}^{2}\left(C_{j} e^{-k_{j} x}-C_{j+3} e^{k_{j} x}\right) .
$$

\section{APPLICATIONS}

When the two ends of the micro-scale beam are clamped, then the boundary conditions are given by

$$
\left.w(x, t)\right|_{x=0, L}=\left.\frac{\partial w(x, t)}{\partial x}\right|_{x=0, L}=0 .
$$




$$
\left[\begin{array}{cccccc}
1 & 1 & 1 & 1 & 1 & 1 \\
e^{-k_{1} L} & e^{-k_{2} L} & e^{-k_{3} L} & e^{k_{1} L} & e^{k_{2} L} & e^{k_{3} L} \\
-k_{1} & -k_{2} & -k_{3} & k_{1} & k_{2} & k_{3} \\
-k_{1} e^{-k_{1} L} & -k_{2} e^{-k_{2} L} & -k_{3} e^{-k_{3} L} & k_{1} e^{k_{1} L} & k_{2} e^{k_{2} L} & k_{3} e^{k_{3} L} \\
H_{1} & H_{2} & H_{3} & H_{1} & H_{2} & H_{3} \\
-k_{1} H_{1} e^{-k_{1} L} & -k_{2} H_{2} e^{-k_{2} L} & -k_{3} H_{3} e^{-k_{3} L} & k_{1} H_{1} e^{k_{1} L} & k_{2} H_{2} e^{k_{2} L} & k_{3} H_{3} e^{k_{3} L}
\end{array}\right]\left\{\begin{array}{l}
C_{1} \\
C_{2} \\
C_{3} \\
C_{4} \\
C_{5} \\
C_{6}
\end{array}\right\}=\left\{\begin{array}{c}
0 \\
0 \\
0 \\
0 \\
\bar{G}(s) \\
-H_{4}
\end{array}\right\} ;
$$

Additionally, we consider the beam is loaded thermally on the boundary $x=0$ as

$$
\theta(x, z, t)=\phi(x, t) \sin (p z)=\theta_{0} f(x, t) \sin (p z) ;
$$

on $x=0$, where $\theta_{0}$ is a constant. Additionally, let us consider that $f(x, t)$ is varying sinusoidal pulse function with time described mathematically as take after

$$
f(x, t)=f(t)=\left\{\begin{array}{ll}
\sin \left(\frac{\pi}{t_{0}} t\right), & 0 \leq t \leq t_{0} \\
0, & t>t_{0}, \quad t<0
\end{array} ;\right.
$$

where $t_{0}$ is a non-negative constant called pulse width parameter. Moreover, the temperature at the end boundary should fulfill the accompanying equation

$$
\left.\frac{\partial \theta(x, t)}{\partial x}\right|_{x=L}=0
$$

After using the dimensionless parameters and Laplace transform, the boundary conditions in Eqs. (43), (44), and (46) take the forms

$$
\begin{gathered}
\left.\bar{w}(x, s)\right|_{x=0, L}=\left.\frac{d \bar{w}(x, s)}{d x}\right|_{x=0, L}=0 ; \\
\left.\bar{\phi}(x, s)\right|_{x=0}=\frac{\pi t_{0}}{\pi^{2}+t_{0}^{2} s^{2}} ;\left.\quad \frac{d \bar{\phi}(x, s)}{d x}\right|_{x=L}=0 .
\end{gathered}
$$

Substituting Eqs. (38), and (39) into the boundary conditions of Eq. (47) yields Eq. (48) (see the top of the page), where $\bar{G}(s)=\frac{\pi t_{0}}{\pi^{2}+t_{0}^{2} s^{2}}-H_{4}$. Solution of the above system of linear equations gives the unknown parameters $C_{j}, j=\overline{1,6}$. This completes the solution of the problem in the Laplace transform domain.

\section{INVERSION OF THE LAPLACE TRANSFORMS (NUMERICAL INVERSION)}

To get a solution of the problem in the physical field, the transforms in Eqs. (38), (39), (41), and (42) are inverted. In order to invert the Laplace transform in the above equations, we adopt a numerical inversion technique based on a Fourier series expansion. ${ }^{35}$ In this technique, the inverse $\Psi(t)$ of the Laplace transform $\bar{\Psi}(s)$ is come close to the relation

$$
\begin{gathered}
\Psi(t)=\frac{e^{c t}}{t_{1}}\left[\frac{1}{2} \bar{\Psi}(c)+\operatorname{Re}\left\{\sum_{k=1}^{n} \bar{\Psi}\left(c+\frac{i k \pi}{t_{1}}\right)\right\}\right] ; \\
0 \leq t \leq t_{1}
\end{gathered}
$$

where $n$ is a sufficiently large integer representing the number of terms in the truncated infinite Fourier series, $n$ may be chosen such that

$$
e^{c t} \operatorname{Re}\left\{e^{\frac{i n \pi t}{t_{1}}} \bar{\Psi}\left(c+\frac{i k \pi}{t_{1}}\right)\right\} \leq \varepsilon_{1}
$$

in which $\varepsilon_{1}$ is a persecuted small positive number that corresponds to the degree of accuracy to be achieved. The parameter $c$ is a positive free parameter that must be larger than the real parts of all singularities of $\bar{\Psi}(s)$. The optimal choice of $c$ was obtained according to the criteria described in Honig and Hirdes. ${ }^{35}$ In order to find the displacement distribution $u(t)$, we use the expression that appeared in Eq. (48) with replacing $\Psi(t)$ and $\bar{\Psi}(s)$ respectively. This procedure is repeated for the other functions.

\section{DISCUSSION OF THE NUMERICAL RESULTS}

Numerical results with the help of Mathematica programming software in the case of silicon nitride have been presented. For this purpose, we take the following values of the different physical constants of the thermoviscoelastic material $\rho=8.954 \times 10^{3} \mathrm{~kg} / \mathrm{m}^{3}, C_{E}=383.1 \mathrm{~J} /(\mathrm{kgK}), T_{0}=296 \mathrm{~K}$, $\alpha_{T}=1.78 \times 10^{-5} \mathrm{~K}^{-1}, \lambda=7.76 \times 10^{10} \mathrm{~kg} / \mathrm{m}^{3}, \mu=$ $3.86 \times 10^{10} \mathrm{~kg} / \mathrm{m}^{3}, K=386 \mathrm{~W} /(\mathrm{mK}), E=8.4 \times 10^{10} \mathrm{~kg} / \mathrm{m}^{3}$, $\alpha_{1}=0.6 \mathrm{~s}, t=0.1 \mathrm{~s}, \alpha_{2}=0.9 \mathrm{~s}, \tau_{q}=0.05 \mathrm{~s}, \tau_{\theta}=0.01 \mathrm{~s}$, $\nu=0.33$.

The aspect ratios of the beam are fixed as $L / h=10$ and $b / h=0.5$. When $h$ is varied, $L$ and $b$ change accordingly with $h$. The figures were prepared by using the non-dimensional variables which are defined in Eq. (22) for a wide range of beam length when $L=1$ and $z=h / 6$. Generally speaking, the value of $R_{a}$ depends on the material of the resonator and the wavelength. For a resonator made of silicon, $R_{a}$ is about 0.17 without coating. With an energy absorbing coating, $R_{a}$ can be larger than 0.99 . Without loss of generality, we take $R_{a}=0.5$ in the following studies. The energy intensity of the laser pulse is $L_{0}=10^{11} \mathrm{~J} / \mathrm{m}^{2}$. For different values of $L_{0}$, which is related to the absorption depth, the beam absorbed different quantity of energy and it vibrates in different manners.

Numerical calculations are carried out for non-dimensional lateral vibration, temperature, displacement and stress along the $x$-direction for various values of the phase lags, different theories of thermoelasticity, pulse width, viscosity, and the time of the laser-pulse and the laser intensity parameters, respectively. Then computations were performed for one value of time, namely for $t=0.1$. The solutions displayed in different figures are observed to be completely in agreement with the boundary conditions under four different cases as follows:

Case I: Discussing the behavior of the field quantities through the axial direction of the micro-scale beam with different values of the phase-lags $\tau_{q}$ and $\tau_{\theta}$ for fixed values of the pulse width parameter $t_{0}=0.2$ and the time of the laser-pulse $t_{p}$, and the laser intensity $L_{0}$ parameters remain constant (see Fig. 2). 


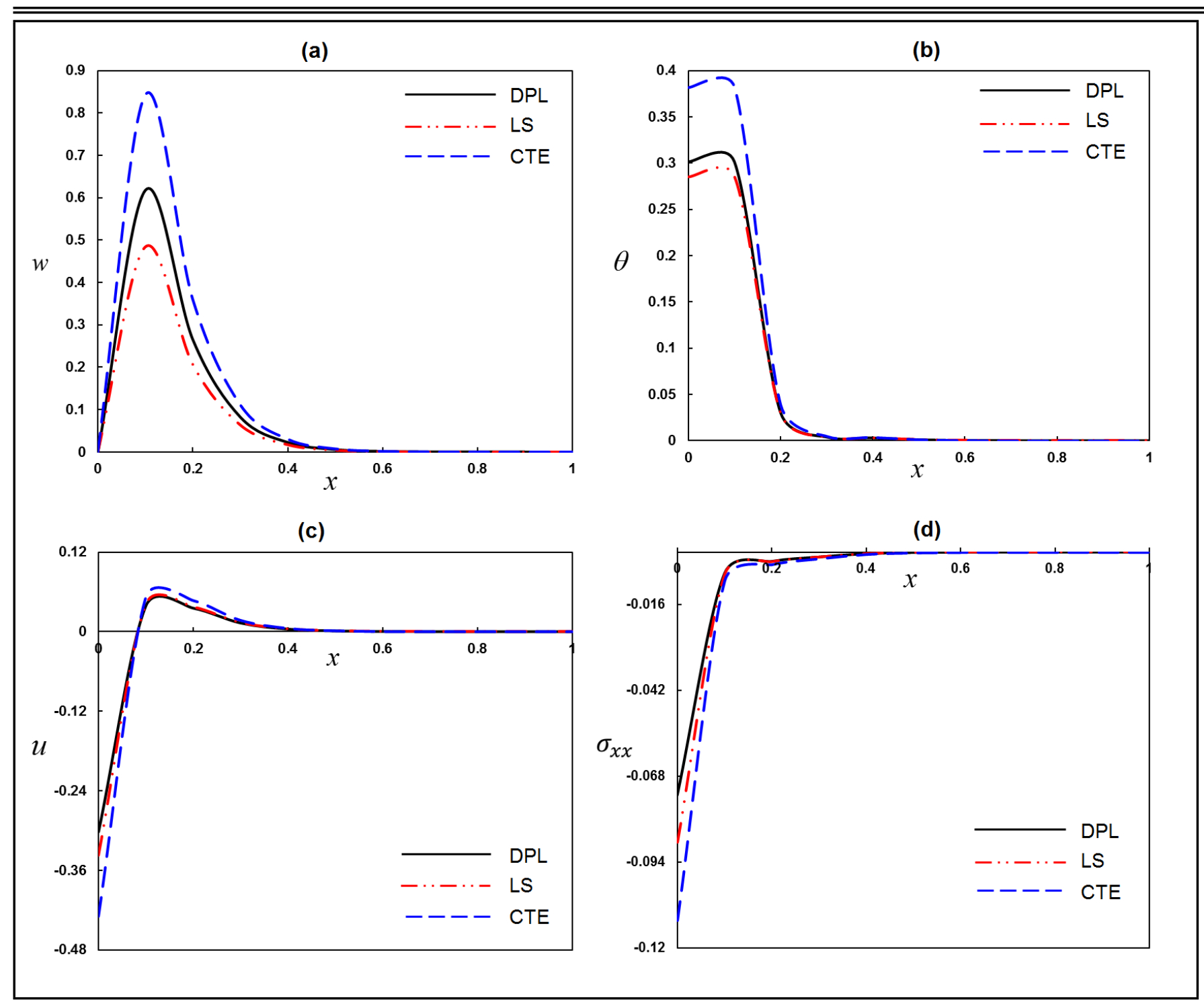

Figure 2. Distributions of the micro-scale beam's: (a) transverse deflection $w$, (b) temperature $\theta$, (c) displacement $u$, and (d) thermal stress $\sigma_{x x}$ versus $x$ for different values of the phase-lags $\tau_{q}$ and $\tau_{\theta}$.

Case II: Investigating how the dimensionless lateral vibration, thermodynamic temperature, displacement, and stress vary with different values of the dimensionless pulse width parameter $t_{0}$ (see Fig. 3 ).

Case III: Introducing the study of the effects of viscosity $\alpha_{1}$ and $\alpha_{2}$ on the dimensionless lateral vibration, thermodynamic temperature, displacement, and stress (see Fig. 4).

Case IV: Studying how the dimensionless lateral vibration, thermodynamic temperature, displacement, and stress vary with different values of the time of the laser-pulse $t_{p}$ and the laser intensity $L_{0}$ parameters (see Fig. 5).

Figures $2 \mathrm{a}$ to $2 \mathrm{~d}$ are drawn to give comparison of the results obtained for displacements, temperature, and stress of the beam against $x$ for different values of phase-lags $\tau_{q}$ and $\tau_{\theta}$ when the pulse width parameter $t_{0}$, the time of the laser-pulse $t_{p}$, and the laser intensity $L_{0}$ parameters remain constant. The graphs in Fig. 2 represent four curves predicted by three different theories of thermoelasticity obtained as a special case of the dual-phase-lag model. The computations were performed for one value of time, namely for $t=0.12$ and various values of the parameters $\tau_{q}$ and $\tau_{\theta}$. These computations were carried out in the coupled theory (CTE) by setting $\tau_{q}=\tau_{\theta}=0$, in Lord-Shulman theory (LS) putting $\tau_{q}>0, \tau_{\theta}=0$, and in the generalized theory of thermoelasticity proposed by Tzou when $\tau_{q} \geq \tau_{\theta}>0$.

Figure 2 a depicts the distribution of the lateral vibration $w$ which always begins from the zero values (i.e., vanishes) and satisfies the boundary condition at $x=0$. Figure $2 \mathrm{~b}$ shows the variant of temperature $\theta$ with $x$ and it indicates that temperature field has maximum value at the boundary $x=0$ of the beam and then falls to zero in the region $0.3 \leq x \leq 1$. The temperature distribution $\theta$ decays along the direction of the transmitted wave propagation for the effects of diffusion. Figure $2 \mathrm{c}$ is plotted to show the variation of thermal displacement $u$ against $x$ for theories of thermoelasticity. It is observed from this figure some difference in values of displacement is noticed for different values of the parameters $\tau_{q}$ and $\tau_{\theta}$. As shown in Fig. 2c, displacement $u$ increases near the micro-scale beam edge $x=0$ (always starts from a negative value), then smooth decreases again to reach its minimum magnitude just at about the beam end. Figure $2 \mathrm{~d}$ displays thermal stress $\sigma_{x x}$. We can see that stress starts from a negative value and terminates at the zero value. It reaches the minimum value nearby the end of the beam and converges to zero with the increasing distance $x$. 
(a)

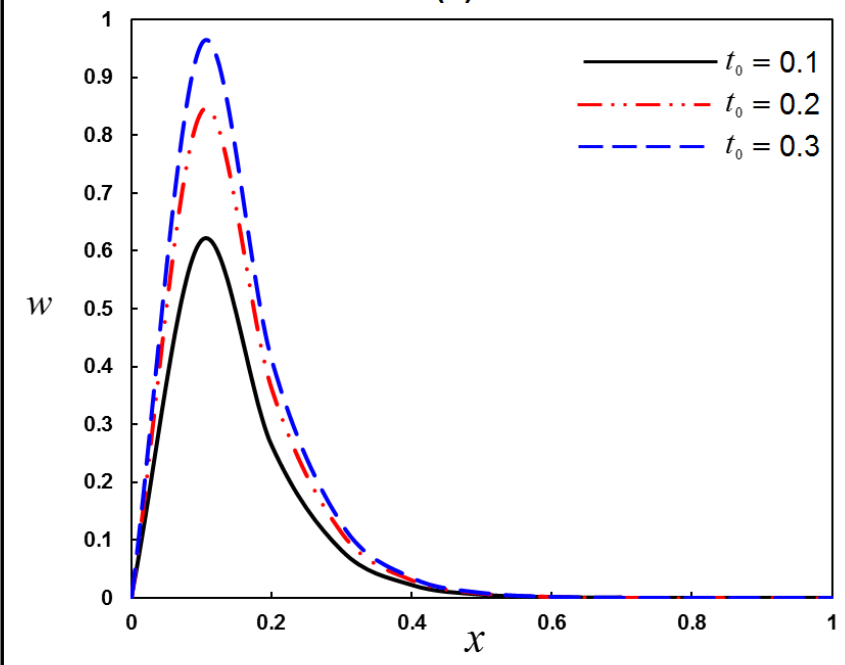

(c)

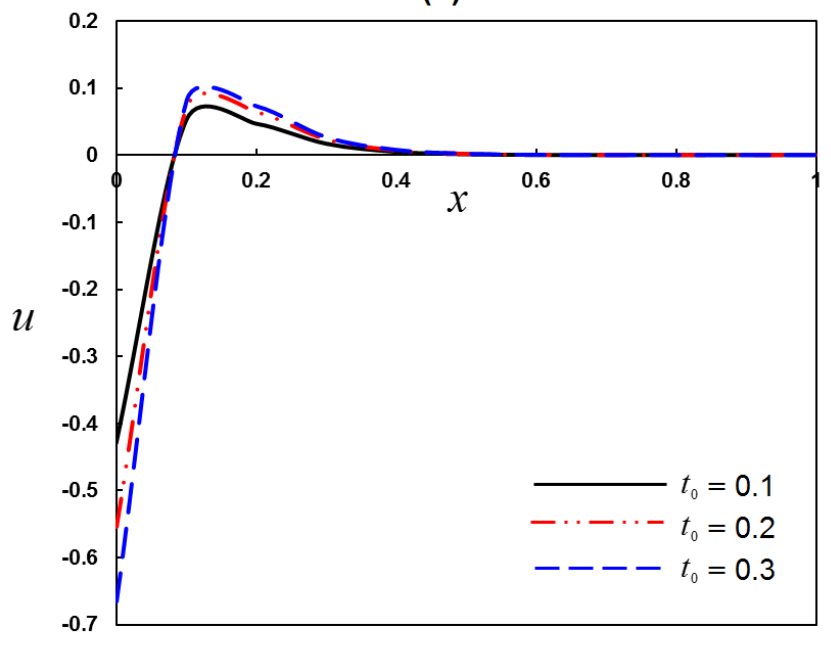

(b)

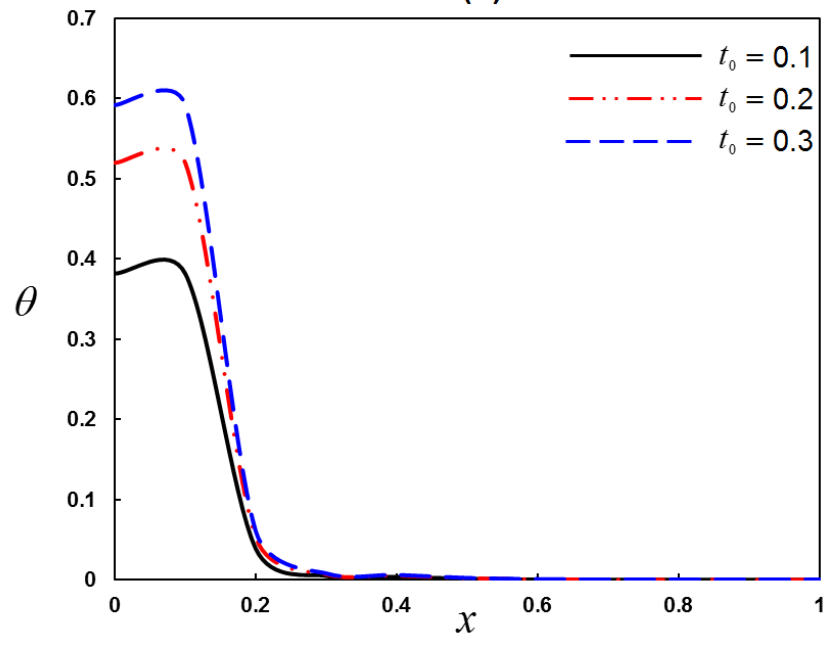

(d)

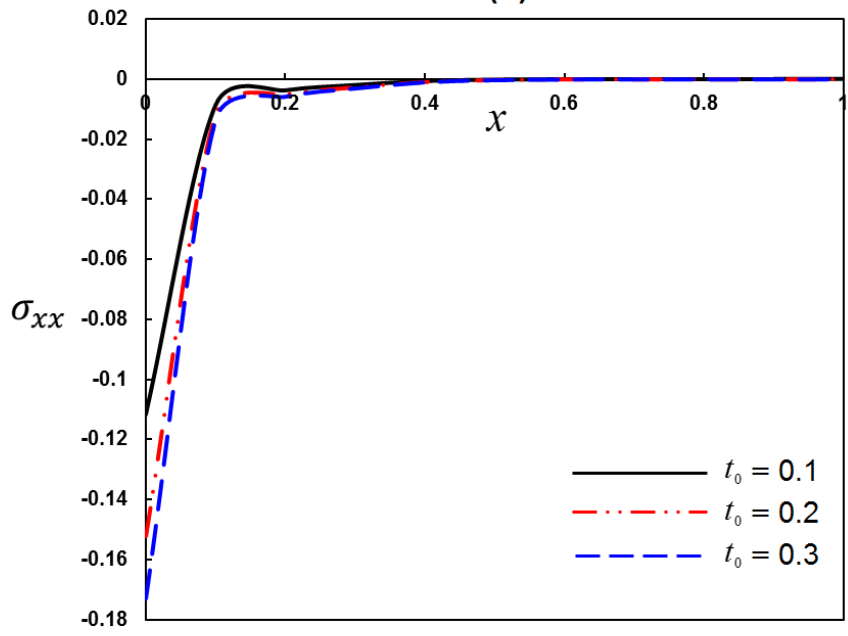

Figure 3. Distributions of the micro-scale beam's: (a) transverse deflection $w$, (b) temperature $\theta$, (c) displacement $u$, and (d) thermal stress $\sigma_{x x}$ versus $x$ for different values of the pulse width parameter $t_{0}$.

It is known that the phase-lag time $\tau_{q}$ can dictate the manners of thermal wave propagation, slow down the propagation speed of thermal indication, and manifest the feature of a thermal wave. The influence of $\tau_{\theta}$ can assist heat energy diffuse and create the characters of thermal wave decline in DPL heat transmission. We found that the phase lags $\tau_{q}$ and $\tau_{\theta}$ parameters have significant effects on all the considered fields. The value of the studied fields for CTE model are larger compared to those for other theories. The distribution in LS theory is close to that in dual phase lag theory, whereas the distributions in the coupled theory CTE is different from that in DPL theory. The phenomenon of a finite speed of propagation is manifested in all figures. This is different from the cases in both the uncoupled and coupled theories of thermoelasticity where an infinite speed of propagation is inherent, and hence all the considered functions have non-zero values for any point in the medium.

The second case is investigating how the non-dimensional lateral vibration, temperature, displacement, and thermal stress vary with pulse width parameter $t_{0}$ when the phas lags $\tau_{q}$ and $\tau_{\theta}$, the the time of the laser-pulse $t_{p}$, and the laser intensity $L_{0}$ parameters remain constants. The numerical results are obtained and presented graphically in Figs. 3a to 3d. We can see the significant effect of the pulse width parameter $t_{0}$ on all the studied fields. The field quantities are very sensitive to the variation of the pulse width parameter $t_{0}$.

In order to study the effects of viscosity on displacement, temperature, stress we now present our results of the numerical evaluation in the form of graphs (Figs. 4a to 4d). It is observed that due to the existence of viscosity term in the phase lag model the amplitude of the thermoelastic fields has significantly decreased for viscous case in comparison with nonviscous case. Due to the presence of viscosity, the magnitude of temperature decreases for all models, and in this case, the magnitude of temperature also approaches zero value with increase of $x$. Additionally, it is observed that the magnitude of displacement and stress distributions is large for viscous case in comparison with non-viscous case.

The last case is studying how the non-dimensional lateral vibration, temperature, displacement and stress vary with different the time of the laser-pulse $t_{p}$ and the laser intensity $L_{0}$ parameters when the phase-lags $\tau_{q}$ and $\tau_{\theta}$ remain constant. The numerical results are obtained and presented graphically in Figs. 5a to 5d. Here, we observe that the significant effect of the time of the laser-pulse and the laser intensity parameters on all the studied fields. The increasing in the value of the laserpulse parameter $t_{p}$ and the laser intensity $L_{0}$ causes decreasing 


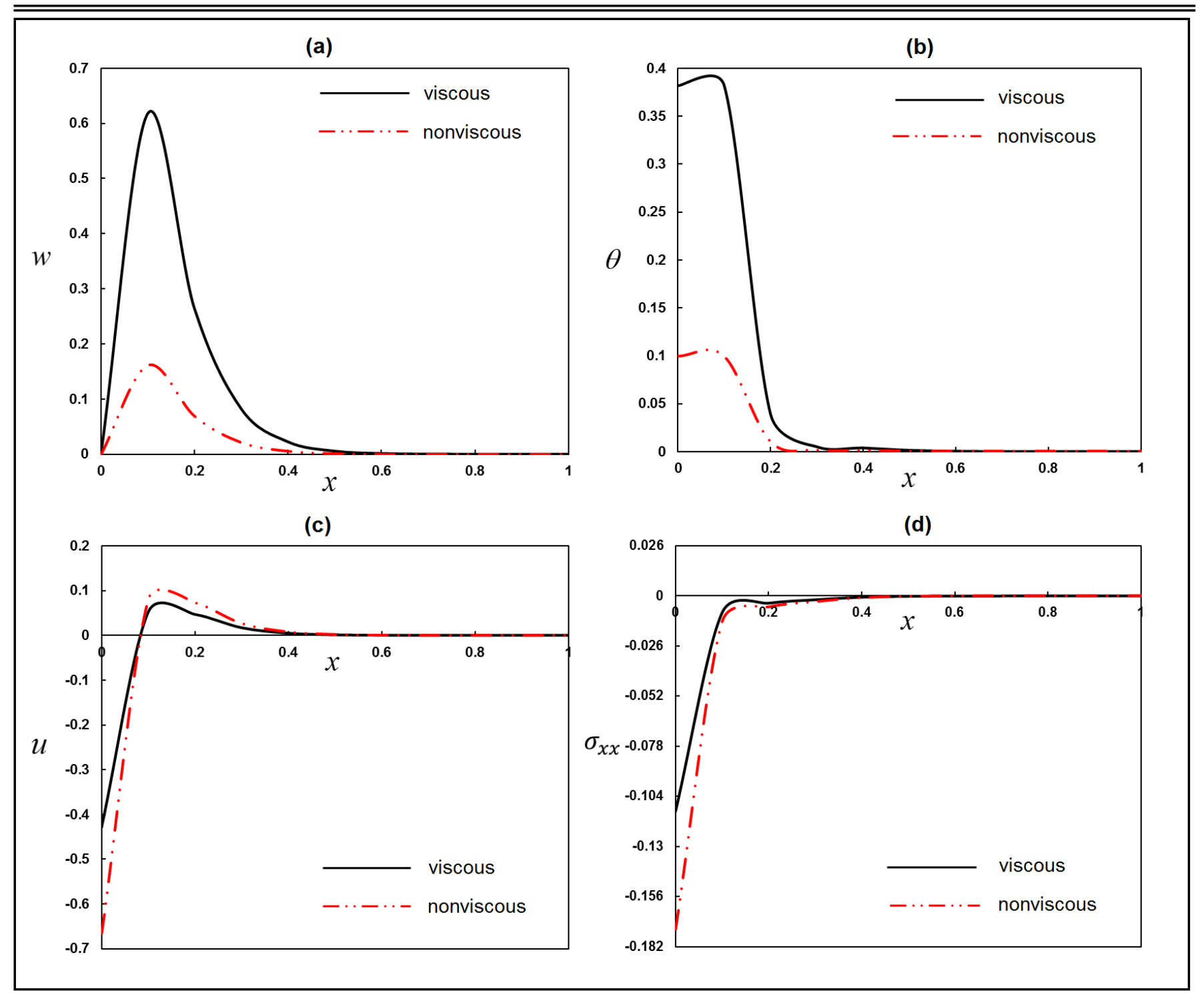

Figure 4. Distributions of: (a) transverse deflection $w$, (b) temperature $\theta$, (c) displacement $u$, and (d) thermal stress $\sigma_{x x}$ versus $x$ for the viscous and nonviscous micro-scale beams.

in the values of all the fields, which is very obvious in the peek points of the curves.

\section{CONCLUSIONS}

In the present study, a generalized thermoviscoelastic problem of Kelvin-Voigt type micro-scale beams subjected to a sinusoidal varying thermal load is considered. The upper surface of the beam is also excited regularly by a laser pulse with a temporal shape in a non-Gaussian form. The phase lag thermoelastic model is applied to study the mechanical relaxations (viscous effects) and thermomechanical coupling (thermal effects). The effects of the phase-lags $\left(\tau_{q}\right.$ and $\left.\tau_{\theta}\right)$ and the pulse width parameters on the field variables were studied. Additionally, the effects of the laser-pulse and the laser intensity parameters of thermal vibration have been studied.

The analysis of the results permits some concluding remarks:

- Visco-thermoelastic resonators provide a useful contribution to thermoelastic coupling in solids in microelectromechanical systems (MEMS) applications.

- It can be observed from the discussion that the phase lags influence plays a significant character on all distributions.

- The difference between coupled theory and dual-phaselag (DPL) model is very clear.

- Due to the existence of viscosity term in the model of thermoelasticity with phase lags, the amplitude of the thermoelastic fields has significantly decreased for viscous case in comparison with non-viscous case.

- Also we found that, the laser-pulse and the laser intensity parameters have significant effects on all the studied fields.

- Lord and Shulman theory (LS) and Green and Naghdi model (GN) as well as the classical thermoelasticity theory (CTE) were obtained as special cases. It was shown that the present DPL model results are close to the LS results.

\section{ACKNOWLEDGEMENTS}

This project was funded by the Deanship of Scientific Research (DSR), King Abdulaziz University, Jeddah, under grant 


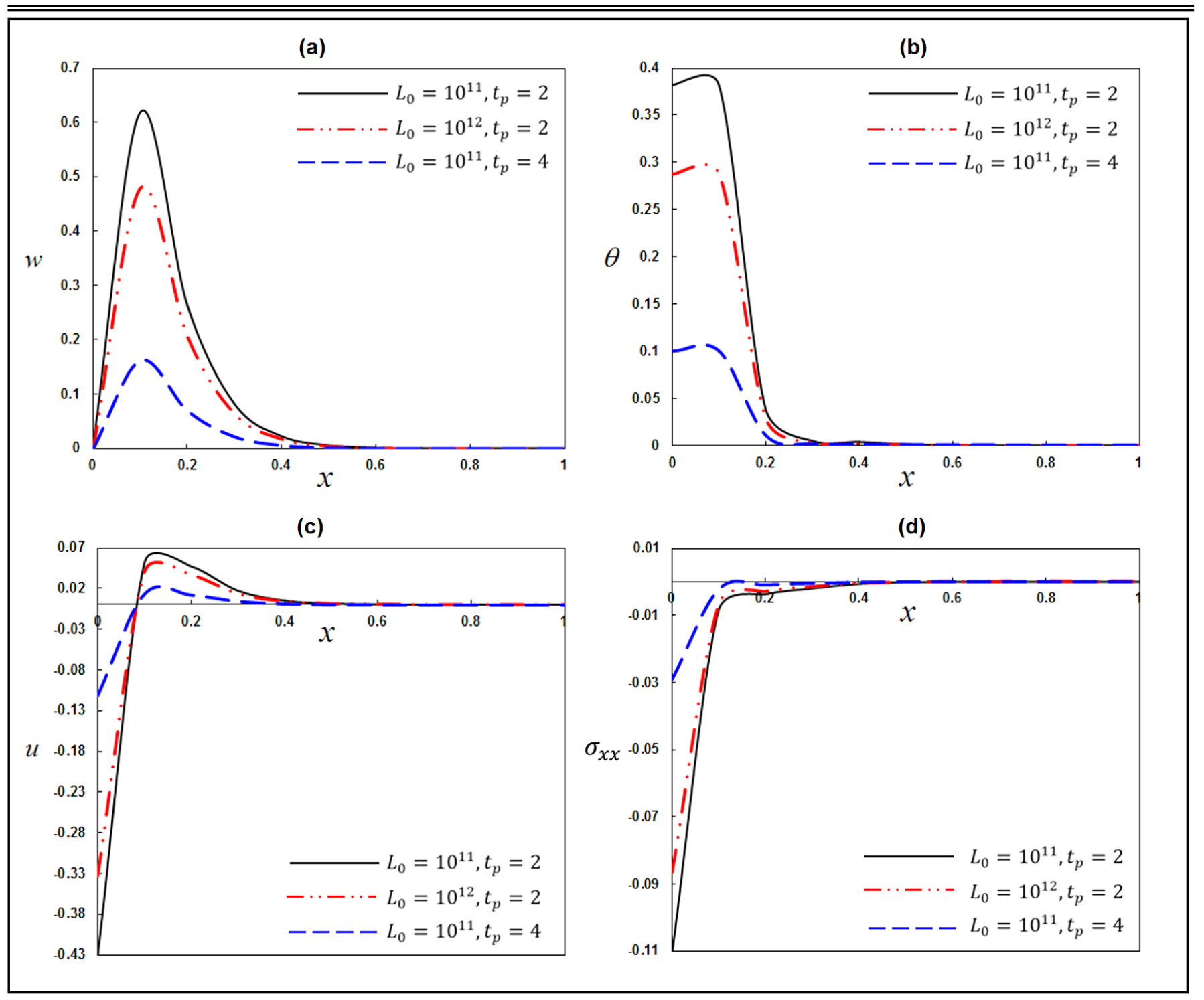

Figure 5. Distributions of the micro-scale beam's: (a) transverse deflection $w$, (b) temperature $\theta$, (c) displacement $u$, and (d) thermal stress $\sigma_{x x}$ versus $x$ for different values of the laser-pulse $t_{p}$ and the laser intensity $L_{0}$ parameters.

no. G-1436-130-195. The authors, therefore, acknowledge with thanks DSR technical and financial support.

\section{REFERENCES}

1 Grover, D. Viscothermoelastic vibrations in micro-scale beam resonators with linearly varying thickness, Canadian Journal of Physics, 90 (5), 487-496, (2012). https://dx.doi.org/10.1139/p2012-044

2 Grover, D. Transverse vibrations in micro-scale viscothermoelastic beam resonators, Archive of Applied Mechanics, 83 (2), 303-314, (2013). https://dx.doi.org/10.1007/s00419-012-0656-y

3 Pal, P., Das, P., and Kanoria, M. Magneto-thermoelastic response in a functionally graded rotating medium due to a periodically varying heat source, Acta Mechanica, 226 (7), 2103-2120, (2015). https://dx.doi.org/10.1007/s00707015-1301-y

4 Zenkour, A. M., Alzahrani, E. O., and Abouelregal, A. E. Generalized magneto-thermoviscoelasticity in a perfectly conducting thermodiffusive medium with a spherical cavity, Journal of Earth System Science, 124 (8), 1709-1719, (2015). https://dx.doi.org/10.1007/s12040-015-0628-z

5 Bucur, A. Rayleigh surface waves problem in linear thermoviscoelasticity with voids, Acta Mechanica, 227 (4), 1199-1212, (2016). https://dx.doi.org/10.1007/s00707$015-1527-8$

6 Ezzat, M. A. and El-Bary, A. A. Unified fractional derivative models of magneto-thermo-viscoelasticity theory, Archives of Mechanics, 68 (4), 285-308, (2016).

7 Doğuşcan Akbaş, Ş. Forced vibration responses of functionally graded viscoelastic beams under thermal environment, International Journal of Innovative Research in Science, Engineering and Technology, 5 (12), 36-46, (2016).

8 Ezzat, M. A. and El-Bary, A. A. On thermo-viscoelastic infinitely long hollow cylinder with variable thermal conductivity, Microsystem Technologies, in press, (2017). https://dx.doi.org/10.1007/s00542-016-3101-2

9 Zenkour, A. M. and Abouelregal, A. E. Effects of phaselags in a thermoviscoelastic orthotropic continuum with a 
cylindrical hole and variable thermal conductivity, Archives of Mechanics, 67 (6), 457-475, (2015).

10 Lord, H. W. and Shulman, Y. A generalized dynamical theory of thermoelasticity, Journal of the Mechanics and Physics of Solids, 15, 299-307, (1967). https://dx.doi.org/10.1016/0022-5096(67)90024-5

11 Green, A. E. and Lindsay, K. A. Thermoelasticity, Journal of Elasticity, 2, 1-7, (1972). https://dx.doi.org/10.1007/BF00045689

12 Dhaliwal, R. S. and Sherief, H. H. Generalized thermoelasticity for anisotropic media, Quarterly of Applied Mathematics, 38 (1), 1-8, (1980). https://dx.doi.org/10.1090/qam/575828

13 Tzou, D. Y. A unified approach for heat conduction from macro-to micro-scales, Journal of Heat Transfer, 117, 816, (1995). https://dx.doi.org/10.1115/1.2822329

14 Tzou, D. Y. Macro- to microscale heat transfer: The lagging behavior, series in chemical and mechanical engineering, Taylor \& Francis, Washington, DC, (1997). https://dx.doi.org/10.1002/9781118818275

15 Sun, H. X. and Zhang, S. Y. Thermoviscoelastic finite element modeling of laser-generated ultrasound in viscoelastic plates, Journal of Applied Physics, 108, 123101, (2010). https://dx.doi.org/10.1063/1.3520675

16 Kumar, R., Kumar, A., and Singh, D. Interaction of laser beam with micropolar thermoelastic solid, Advances in Physics Theories and Applications, 40, 10-15, (2015).

17 Scruby, C. and Drain, L. Laser ultrasonics: Techniques and applications, Adam Hilger, New York, (1990).

18 Royer, D. Mixed matrix formulation for the analysis of laser-generated acoustic waves by a thermoelastic line source, Ultrasonics, 39, 345-354, (2001). https://dx.doi.org/10.1016/S0041-624X(01)00066-X

19 Wang, X. and Xu, X. Thermoelastic wave induced by pulsed laser heating, Applied Physics A, 73, 107-114, (2001). https://dx.doi.org/10.1007/s003390000593

20 Wang, $\mathrm{X}$. and $\mathrm{Xu}, \mathrm{X}$. Thermoelastic wave in metal induced by ultrafast laser pulses, Journal of Thermal Stresses, 25, 457-473, (2002). https://dx.doi.org/10.1080/01495730252890186

21 Allam, M. N. M. and Abouelregal, A. E. The thermoelastic waves induced by pulsed laser and varying heat of inhomogeneous microscale beam resonators, Journal of Thermal Stresses, 37 (4), 455-470, (2014). https://dx.doi.org/10.1080/01495739.2013.870858

$22 \mathrm{Fu}, \mathrm{Y}$. and Zhang, J. Free vibration analysis of a micro-scale beam subjected to a symmetric electrostatic field, Mechanics of Advanced Materials and Structures, 20 (4), 257-263, (2013). https://dx.doi.org/10.1080/15376494.2011.584264

23 Zenkour, A. M. and Abouelregal, A. E. Effect of harmonically varying heat on FG nanobeams in the context of a nonlocal two-temperature thermoelasticity theory, European Journal of Computational Mechanics, 23 (1-2), 1-14, (2014). https://dx.doi.org/10.1080/17797179.2014.882141
${ }^{24}$ Carrera, E., Abouelregal, A. E., Abbas, I. A., and Zenkour, A. M. Vibrational analysis for an axially moving micro-scale beam with two temperatures, Journal of Thermal Stresses, 38, 569-590, (2015). https://dx.doi.org/10.1080/01495739.2015.1015837

25 Abouelregal, A. E. and Zenkour, A. M. Thermoelastic problem of an axially moving micro-scale beam subjected to an external transverse excitation, Journal of Theoretical and Applied Mechanics, 53 (1), 167-178, (2015). https://dx.doi.org/10.15632/jtam-pl.53.1.167

26 Abouelregal, A. E. and Zenkour, A. M. Effect of phase lags on thermoelastic functionally graded micro-scale beams subjected to ramp-type heating, IJST, Transactions of Mechanical Engineering, 38 (M2), 321-335, (2014).

27 Abouelregal, A. E. and Zenkour, A. M. Generalized thermoelastic vibration of a micro-scale beam with an axial force, Microsystem Technologies, 21 (7), 1427-1435, (2015). https://dx.doi.org/10.1007/s00542-014-2220-x

28 Zenkour, A. M. Free vibration of a micro-scale beam resting on Pasternak's foundation via the GN thermoelasticity theory without energy dissipation, Low Frequency Noise, Vibration and Active Control, 35 (4), 303-311, (2016).

29 Zenkour, A. M. and Abouelregal, A. E. Thermoelastic vibration of an axially moving micro-scale beam subjected to sinusoidal pulse heating, International Journal of Structural Stability and Dynamics, 15 (6), 1450081, (2015). https://dx.doi.org/10.1142/S0219455414500813

30 Zenkour, A. M. and Abouelregal, A. E. The effect of two temperatures on a FG nanobeam induced by a sinusoidal pulse heating, Structural Engineering and Mechanics, 51 (2), 199-214, (2014). https://dx.doi.org/10.12989/sem.2014.51.2.019

31 Zenkour, A. M. and Abouelregal, A. E. Nonlocal thermoelastic nanobeam subjected to a sinusoidal pulse heating and temperature-dependent physical properties, $\mathrm{Mi}$ crosystem Technologies, 21 (8), 1767-1776, (2015). https://dx.doi.org/10.1007/s00542-014-2294-5

32 Mishra, J. C., Samanta, S. C., and Chakrabarty, A. K. Magneto-thermo-mechanical interaction in an aeolotropic viscoelastic cylinder permeated by magnetic field subjected to a periodic loading, International Journal of Engineering Science, 29, 1209-1216, (1991). https://dx.doi.org/10.1016/0020-7225(91)90025-X

33 Mukhopadhyay, S. Effects of thermal relaxations on thermoviscoelastic interactions in an unbounded body with a spherical cavity subjected to a periodic loading on the boundary, Journal of Thermal Stresses, 23, 675-684, (2000). https://dx.doi.org/10.1080/01495730050130057

34 Boley, B. A. Approximate analyses of thermally induced vibrations of beams and plates, Journal of Applied Mechanics, 39, 212-216, (1972). https://dx.doi.org/10.1115/1.3422615

35 Honig, G. and Hirdes, U. A method for the numerical inversion of the Laplace transform, Journal of Computational and Applied Mathematics, 10, 113-132, (1984). https://dx.doi.org/10.1016/0377-0427(84)90075-X 